\title{
PHASE PORTRAITS OF CONTINUOUS PIECEWISE LINEAR LIÉNARD DIFFERENTIAL SYSTEMS WITH THREE ZONES
}

\author{
SHIMIN LI ${ }^{1}$ AND JAUME LLIBRE ${ }^{2}$
}

\begin{abstract}
This paper deals with a class of planar continuous piecewise linear Liénard differential systems with three zones separated by two vertical lines without symmetry. We provide the topological classification of the phase portraits in the Poincaré disc for systems having a unique singular point located in the middle zone.
\end{abstract}

\section{INTRODUCTION AND MAIN RESULTS}

In recent years there is a growing interest in the analysis of planar continuous piecewise linear Liénard differential systems of the form:

$$
\frac{d x}{d t}=y-f(x), \quad \frac{d y}{d t}=a-g(x),
$$

where

$$
f(x)= \begin{cases}k_{1}(x-1)+k_{2} & \text { if } x \geqslant 1, \\ k_{2} x & \text { if }-1 \leqslant x \leqslant 1, \\ k_{3}(x+1)-k_{2} & \text { if } x \leqslant-1,\end{cases}
$$

and

$$
g(x)= \begin{cases}l_{1}(x-1)+l_{2} & \text { if } x \geqslant 1, \\ l_{2} x & \text { if }-1 \leqslant x \leqslant 1, \\ l_{3}(x+1)-l_{2} & \text { if } x \leqslant-1 .\end{cases}
$$

Systems (1) satisfying (2) and (3) have been studied extensively, see for instance $[7,9,10,14,15,17,21]$. For the symmetrical cases, that is $a=0, k_{1}=k_{3}$ and $l_{1}=l_{3}$, see [9] for the bifurcation sets and existence of the limit cycles, see [10] for the amplitude and period of the limit cycles, see [17] for the global phase portraits and bifurcation diagrams. While for the non-symmetrical case, the analysis of systems (1) satisfying (2) and (3) become more complicated. In [14] it is considered the existence and uniqueness of limit cycles for the case $k_{1}>0, k_{1}<0, k_{3}>0$. In [15] the authors studied the uniqueness and non-uniqueness of limit cycles for the more general cases, they showed that systems (1) satisfying (2) and (3) can have at leat two limit cycles for the cases either $k_{1}>0, k_{2}<0, k_{3}<0$, or $k_{1}<0, k_{2}<$

2010 Mathematics Subject Classification. 34A36, 34C07, 37G05.

Key words and phrases. Limit cycle; Piecewise linear differential system; Phase portrait; Liénard system. 
$0, k_{3}>0$. In [7] the authors found that systems (1) satisfying (2) and (3) can exist jump bifurcations when $l_{2}=0$. In [21] it is investigated the boundary equilibrium bifurcations of systems (1) satisfying (2) and (3).

It is worth to note that if either $k_{1}=k_{2}, l_{1}=l_{2}$, or $k_{2}=k_{3}, l_{2}=l_{3}$, then systems (1) satisfying (2) and (3) become continuous piecewise linear Liénard differential systems with two zones. In 1990 Lum and Chua $[18,19]$ conjectured that continuous piecewise linear differential systems with two zones separated by a straight line have at most one limit cycle. In 1998 Freire et al. [8] gave a positive answer to this conjecture. Note that this conjecture cannot be extended to discontinuous piecewise linear differential systems see $[1,12]$, and to continuous piecewise linear differential systems with non-regular separation line see $[3,16]$.

In this paper we study the global phase portraits of systems (1) satisfying (2) and (3). Since the number of different phase portraits is high, in the present paper we only consider the special case $g(x)=x$, that is

$$
\frac{d x}{d t}=y-f(x), \quad \frac{d y}{d t}=a-x,
$$

with $-1<a<1, k_{1} k_{2} k_{3} \neq 0$ and $f(x)$ given in (2). Without loss of generality we assume that $k_{2}<0$. Otherwise we can do the change $X=x, Y=-y, T=-t$, and then obtain $k_{2}<0$.

For polynomial differential systems it is well known that the separatrices in the Poincaré disc are all the infinite orbits, all the finite singular points, the separatrices of the hyperbolic sectors of the finite and infinite singular points, and the limit cycles. If $\Sigma$ denote the set of all separatrices in the Poincaré disk $\mathbb{D}^{2}, \Sigma$ is a closed set and the components of $\mathbb{D}^{2} \backslash \Sigma$ are called the canonical regions. See subsection 2.1 for the definition of the Poincaré compactification, there it is defined the Poincaré $\operatorname{disc} \mathbb{D}^{2}=\left\{\left(s_{1}, s_{2}\right): s_{1}^{2}+s_{2}^{2} \leqslant 1\right\}$. We denote by $\mathrm{S}$ and $\mathrm{R}$ the number of separatrices and canonical regions of a given phase portrait in the Poincaré disc, respectively.

We say that two phase portraits $P_{1}$ and $P_{2}$ of systems (4) are topologically equivalent if there exists a homeomorphism $h: \mathbb{D}^{2} \rightarrow \mathbb{D}^{2}$ such that $h\left(\partial \mathbb{D}^{2}\right)=\partial \mathbb{D}^{2}$, $h\left(\operatorname{int}\left(\mathbb{D}^{2}\right)\right)=\operatorname{int}\left(\mathbb{D}^{2}\right)$, and $h$ maps orbits of $P_{1}$ into orbits of $P_{2}$ either preserving all the orientations, or reversing all the orientations of the orbits. Here $\partial \mathbb{D}^{2}$ and $\operatorname{int}\left(\mathbb{D}^{2}\right)$ denote the boundary and interior of $\mathbb{D}^{2}$, respectively.

It it convenient to introduce the following crucial parameters.

$$
\Delta_{i}=k_{i}^{2}-4, \quad i=1,2,3 .
$$

In section 3 we will see that the type and stability of the finite and infinite singular points of systems (4) satisfying (2) are determined by the signs of $\Delta_{i}$ and $k_{i}$ respectively.

We classify all the topological equivalent phase portraits in the Poincaré disc for systems (4) satisfying (2) according to the sign of $k_{i}$. Our main results are the following three theorems.

Theorem 1. The phase portrait on the Poincaré disc of a continuous piecewise Liénard differential systems (4) satisfying (2) with $-1<a<1$ and $k_{1}<0, k_{2}<$ $0, k_{3}<0$, is topologically equivalent to one of the 6 phase portraits described in Figure 1. 
PHASE PORTRAITS OF CONTINUOUS PIECEWISE LINEAR DIFFERENTIAL SYSTEMS 3

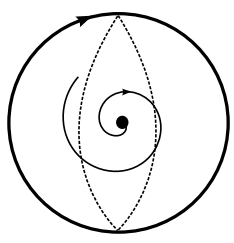

$1.1 \mathrm{~S}=2, \mathrm{R}=1$

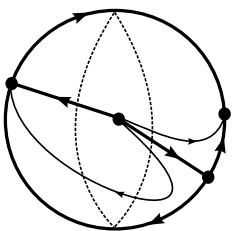

$1.5 S=9, R=2$

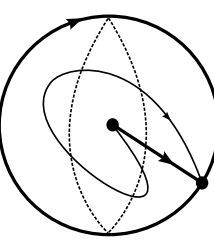

$1.2 S=4, R=1$

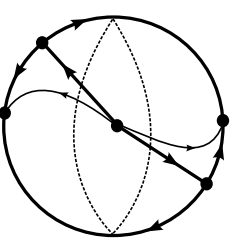

$1.6 \mathrm{~S}=11, \mathrm{R}=2$

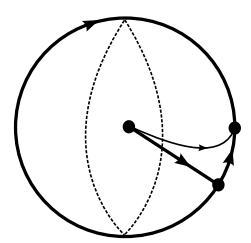

$1.3 \mathrm{~S}=6, \mathrm{R}=1$

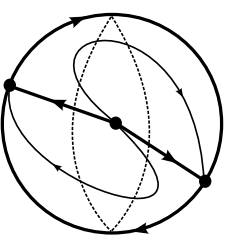

$1.4 S=7, R=2$

FIGURE 1. Topological phase portraits of systems (4) satisfying (2) with $k_{1}<0, k_{2}<0$ and $k_{3}<0$. The right dashed line represents the straight line of $x=1$, and the left dashed curve represents the straight line of $x=-1$.

Theorem 2. The phase portrait on the Poincaré disc of a continuous piecewise Liénard differential systems (4) satisfying (2) with $-1<a<1$ and $k_{1}>0, k_{2}<$ $0, k_{3}>0$, is topologically equivalent to one of the 6 phase portraits described in Figure 2.

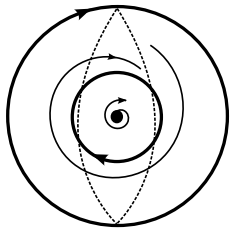

$2.1 S=3, R=2$

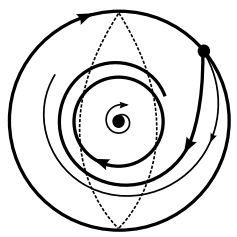

$2.2 S=5, R=2$

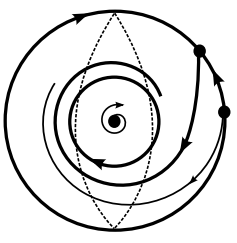

$2.3 S=7, R=2$

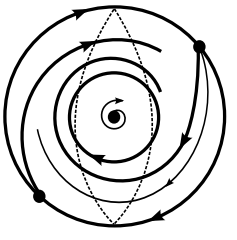

$2.4 \mathrm{~S}=8, \mathrm{R}=2$

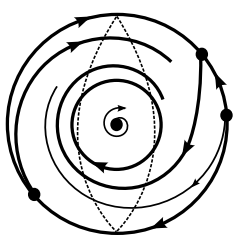

$2.5 \mathrm{~S}=10, \mathrm{R}=2$

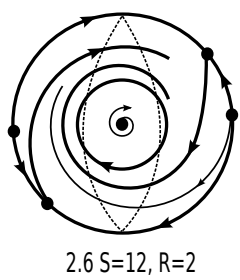

FIgURE 2. Topological phase portraits of systems (4) satisfying (2) with $k_{1}>0, k_{2}<0$ and $k_{3}>0$.

Theorem 3. The phase portrait on the Poincaré disc of a continuous piecewise Liénard differential systems (4) satisfying (2) with $-1<a<1$ and either $k_{1}>$ $0, k_{2}<0, k_{3}<0$, or $k_{1}<0, k_{2}<0, k_{3}>0$, is topologically equivalent to one of the 20 phase portraits described in Figure 3. 


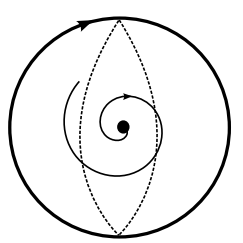

$3.1 \mathrm{~S}=2, \mathrm{R}=1$

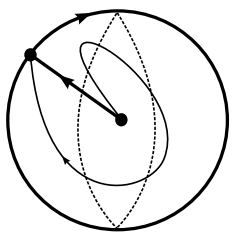

$3.5 \mathrm{~S}=4, \mathrm{R}=1$

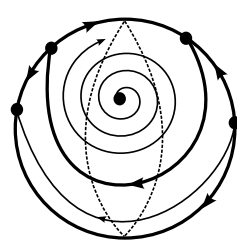

$3.9 \mathrm{~S}=10, \mathrm{R}=2$

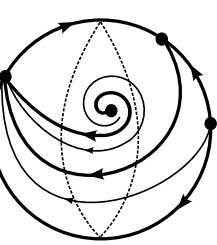

$3.13 S=9, R=2$

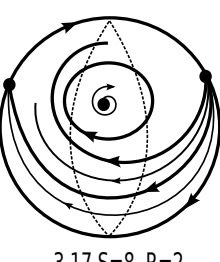

$3.17 \mathrm{~S}=8, \mathrm{R}=2$

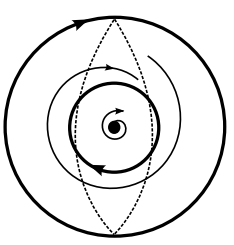

$3.2 S=3, R=2$

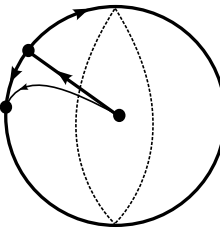

$3.6 \mathrm{~S}=6, \mathrm{R}=1$
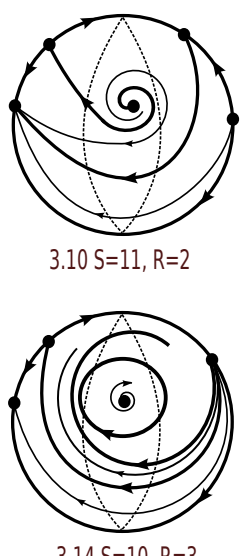

$3.14 \mathrm{~S}=10, \mathrm{R}=3$

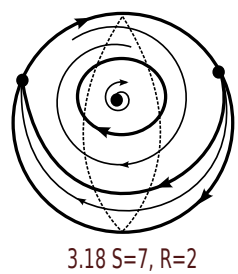

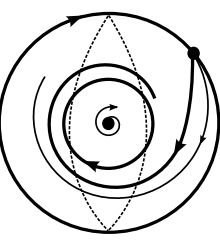

$3.3 S=5, R=2$

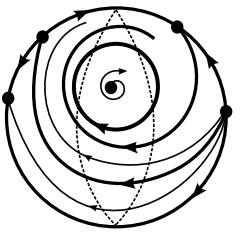

$3.7 S=12, R=3$

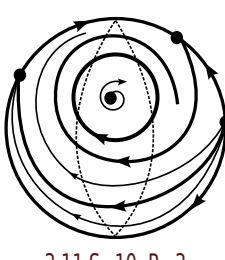

3.11 $S=10, R=3$

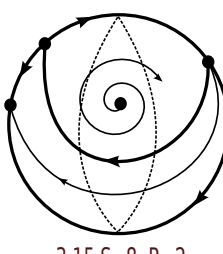

$3.15 \mathrm{~S}=8, \mathrm{R}=2$
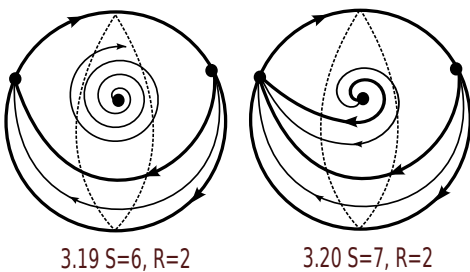

FIgURE 3. Topological phase portraits of systems (4) satisfying (2) with $k_{1}>0, k_{2}<0$ and $k_{3}<0$.

Doing the change of variables $X=\frac{x}{\sqrt{\varepsilon}}, Y=y, T=\frac{t}{\sqrt{\varepsilon}}$, systems (4) satisfying (2) become continuous piecewise linear slow fast Liénard differential systems of the form

(6)

$$
\frac{d X}{d T}=Y-F(X), \quad \frac{d Y}{d T}=\varepsilon(A-X),
$$

where $A=a / \sqrt{\varepsilon}$ and $F(X)=f(\sqrt{\varepsilon} X)$. 
In [13] the authors studied systems (6) with $k_{2}=-1$ and $k_{1}=k_{3}$ a fixed constant greater than 1 , but close to 1 . They proved that systems (6) have at most one limit cycle using first integrals, and also shown that an usual relaxation oscillation occurs for such systems. In [4] it is investigated systems (6) with $k_{1}=k_{3}>0$ and $k_{2}<0$. They found that this kind of differential systems admit limit cycles that share a lot of similarity with the van der Pol canards. In [5] the authors analyzed systems (6) that display fold singularities, primary and secondary canards.

This paper is organized as follows. In section 2 we introduce the Poincaré compactification, which is important for studying the infinite singular points. Section 3 is devoted to investigate the existence and uniqueness of limit cycles. In section 4 we prove our main results by careful qualitative analysis. The conclusions on this work are given in section 5 .

\section{Singular POINTS}

We use Poincaré compactification to analyze the behaviour of finite and infinite singular points, see more details in chapter 5 of [6].

2.1. Poincaré compactification. For a given polynomial differential systems

$$
\frac{d x_{1}}{d t}=P\left(x_{1}, x_{2}\right), \quad \frac{d x_{2}}{d t}=Q\left(x_{1}, x_{2}\right),
$$

of degree $d=\max \{\operatorname{deg}(P), \operatorname{deg}(Q)\}$ in $\mathbb{R}^{2}$. Let $\mathcal{X}=(P, Q)$ the vector field associate systems (7).

First we consider $\mathbb{R}^{2}$ as the plane in $\mathbb{R}^{3}$ defined by $\left(s_{1}, s_{2}, s_{3}\right)=\left(x_{1}, x_{2}, 1\right)$. We call the unit sphere $\mathbb{S}^{2}=\left\{\left(s_{1}, s_{2}, s_{3}\right): s_{1}^{2}+s_{2}^{2}+s_{3}^{2}=1\right\}$ the Poincaré sphere. The equator $\mathbb{S}^{1}=\left\{\left(s_{1}, s_{2}, s_{3}\right) \in \mathbb{S}^{2}, s_{3}=0\right\}$ divide $\mathbb{S}^{2}$ into two parts: the northern hemisphere $\mathbb{S}^{-}=\left\{\left(s_{1}, s_{2}, s_{3}\right) \in \mathbb{S}^{2}: s_{3}>0\right\}$, and the southern hemisphere $\mathbb{S}^{+}=$ $\left\{\left(s_{1}, s_{2}, s_{3}\right) \in \mathbb{S}^{2}: s_{3}<0\right\}$. Now we project each point $\left(x_{1}, x_{2}, 1\right) \in \mathbb{R}^{3}$ onto the Poincaré sphere using a straight line through $\left(x_{1}, x_{2}, 1\right)$ and the origin, it is obvious that the equator $\mathbb{S}^{1}$ corresponds to the infinity of $\mathbb{R}^{2}$. So we have two copies of the vector field $\mathcal{X}$ on the poincaré sphere $\mathbb{S}^{2}$, one in the open northern hemisphere and the other in the open southern hemisphere. This vector field on $\mathbb{S}^{2} \backslash \mathbb{S}^{1}$ can be extended to a vector field $p(\mathcal{X})$ defined in the whole $\mathbb{S}^{2}$ multiplying it by $s_{3}^{d}$.

For studying the Poincaré sphere we consider the local charts

$$
U_{i}=\left\{\mathbf{s} \in \mathbb{S}^{2}: s_{i}>0\right\}, V_{i}=\left\{\mathbf{s} \in \mathbb{S}^{2}: s_{i}<0\right\},
$$

where $\mathbf{s}=\left(s_{1}, s_{2}, s_{3}\right)$.

The expression of $p(\mathcal{X})$ in the local chart $U_{1}$ is given by

$$
\frac{d u}{d t}=v^{d}\left[-u P\left(\frac{1}{v}, \frac{u}{v}\right)+Q\left(\frac{1}{v}, \frac{u}{v}\right)\right], \quad \frac{d v}{d t}=-v^{d+1} P\left(\frac{1}{v}, \frac{u}{v}\right)
$$

with $v>0$.

The expression of $p(\mathcal{X})$ in the local chart $V_{1}$ is also given by $(9)$ with $v<0$. 
The expression of $p(\mathcal{X})$ in the local chart $U_{2}$ is given by

$$
\frac{d u}{d t}=v^{d}\left[-u P\left(\frac{u}{v}, \frac{1}{v}\right)-u Q\left(\frac{u}{v}, \frac{1}{v}\right)\right], \quad \frac{d v}{d t}=-v^{d+1} Q\left(\frac{u}{v}, \frac{1}{v}\right) ;
$$

with $v>0$.

The expression of $p(\mathcal{X})$ in the local chart $V_{2}$ is also given by (10) with $v<0$.

The expression of $p(\mathcal{X})$ in the local charts $U_{3}$ and $V_{3}$ are just

$$
\frac{d u}{d t}=P(u, v), \quad \frac{d v}{d t}=Q(u, v) .
$$

It is clear that to study the phase portrait of the differential systems (7), it suffices to study its Poincaré compactification $p(\mathcal{X})$ restricted to the northern hemisphere. For drawing the phase portraits we do the orthogonal projection $\pi\left(s_{1}, s_{2}, s_{3}\right)=$ $\left(s_{1}, s_{2}\right)$ of the closed northern hemisphere onto the closed unit disc centered at the origin of coordinates in the plane $x_{3}=0$. This closed disc is called the Poincaré $\operatorname{disc} \mathbb{D}^{2}$.

The finite singular points of systems (7) are the singular points in the interior of $\mathbb{D}^{2}$, and they can be studied using $U_{3}$. The infinite singular points of systems $(7)$ are the singular points of $p(\mathcal{X})$ in the boundary of $\mathbb{D}^{2}$. Note that for studying the infinite singular points it suffices to look the ones at the local charts $\left.U_{1}\right|_{v=0},\left.V_{1}\right|_{v=0}$, and at the origin of the local chart $U_{2}$.

2.2. Chart $\mathbf{U}_{1}$. Let $x=\frac{1}{v}, y=\frac{u}{v}, v>0$, then systems (4) satisfying (2) become

$$
\frac{d u}{d t}=a v-1-u^{2}+k_{1} u-k_{1} u v+k_{2} u v, \quad \frac{d v}{d t}=v\left(k_{1}-k_{1} v+k_{2} v-u\right),
$$

with $0<v \leqslant 1$, and

$$
\frac{d u}{d t}=a v-1-u^{2}+k_{2} u, \quad \frac{d v}{d t}=v\left(k_{2}-u\right),
$$

with $v \geqslant 1$.

In order to study the infinite singular points of systems (4) satisfying (2) in chart $U_{1}$, we impose that $v=0$. It is obvious that systems (13) have no singular points because $v \geqslant 1$. Let $v=0$, then the singular points of systems (12) should satisfy $u^{2}-k_{1} u+1=0$. Recall that $\Delta_{1}=k_{1}^{2}-4$, then we have:

Proposition 4. For systems (12) the following statements hold.

(I) If $\Delta_{1}<0$, then systems (12) have no infinite singular points.

(II) If $\Delta_{1}=0$, then systems (12) have one infinite singular point $E_{1}=\left(\frac{k_{1}}{2}, 0\right)$, and it is a saddle-node, see Figure 4.

(III) If $\Delta_{1}>0$, then systems (12) have two infinite singular points: $E_{1}^{+}=$ $\left(\frac{k_{1}+\sqrt{\Delta_{1}}}{2}, 0\right)$, which is a saddle for $k_{1}>0$, and a stable node for $k_{1}<0$; $E_{1}^{-}=\left(\frac{k_{1}-\sqrt{\Delta_{1}}}{2}, 0\right)$, which is an unstable node for $k_{1}>0$, and a saddle for $k_{1}<0$. 


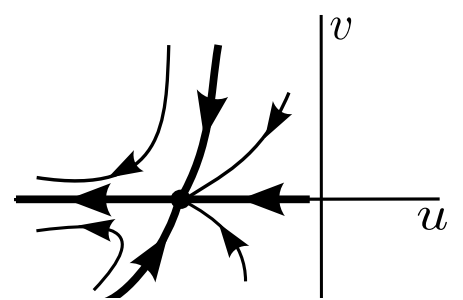

4.1 $k_{1}<0$

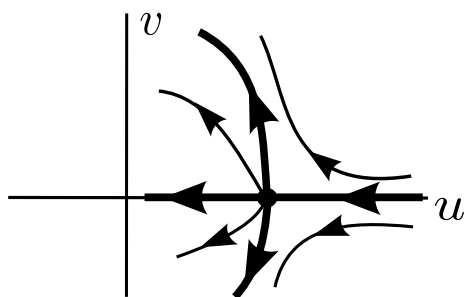

$4.2 k_{1}>0$

Figure 4. The local phase portrait of the saddle-node singular point $E_{1}$ of systems (12).

Proof. (I) Since the infinite singular points must satisfy the equation $u^{2}-k_{1} u+1=$ 0 , statement (I) is obvious.

(II) Since $\Delta_{1}=k_{1}^{2}-4=0$, the Jacobian matrix at the singular point $E_{1}=$ $\left(\frac{k_{1}}{2}, 0\right)$ is

$$
\left(\begin{array}{cc}
0 & a+\frac{k_{1}\left(k_{2}-k_{1}\right)}{0} \\
0 & \frac{k_{1}^{2}}{2}
\end{array}\right) .
$$

We first consider the case $k_{1}=-2$, we do the change $U=1+u-\left(2+k_{2}\right) v, V=$ $v, T=-t$, then systems (12) become

$$
\frac{d U}{d T}=U^{2}-a U V, \quad \frac{d V}{d T}=V(1+U-a V) .
$$

According with Theorem 2.19 of [6] we can deduce that $E_{1}$ is the saddle-node singular point described in Fig. 4.1. Note that we have reverse the time, so the direction is the converse.

The phase portraits of $E_{1}$ described in Fig. 4.2 can be analyzed similarly for the case $k_{1}=2$.

(III) The Jacobian matrix at the singular point $E_{1}^{+}=\left(\frac{k_{1}+\sqrt{\Delta_{1}}}{2}, 0\right)$ is

$$
\left(\begin{array}{cc}
-\sqrt{\Delta_{1}} & a-\left(k_{1}-k_{2}\right) \frac{k_{1}+\sqrt{\Delta_{1}}}{2} \\
0 & \frac{k_{1}-\sqrt{\Delta_{1}}}{2}
\end{array}\right) \text {. }
$$

If $k_{1}>0$, then $E_{1}^{+}$is a saddle. If $k_{1}<0$, then $E_{1}^{+}$is a stable node.

The Jacobian matrix at the singular point $E_{1}^{-}=\left(\frac{k_{1}-\sqrt{\Delta_{1}}}{2}, 0\right)$ is

$$
\left(\begin{array}{cc}
\sqrt{\Delta_{1}} & a-\left(k_{1}-k_{2}\right) \frac{k_{1}-\sqrt{\Delta_{1}}}{2} \\
0 & \frac{k_{1}+\sqrt{\Delta_{1}}}{2}
\end{array}\right) \text {. }
$$

If $k_{1}>0$, then $E_{1}^{-}$is an unstable node. If $k_{1}<0$, then $E_{1}^{-}$is a saddle. 
2.3. Chart $\mathbf{V}_{1}$. Let $x=\frac{1}{v}, y=\frac{u}{v}, v<0$, then systems (4) satisfying (2) become (18) $\frac{d u}{d t}=a v-1-u^{2}+k_{3} u-k_{2} u v+k_{3} u v, \quad \frac{d v}{d t}=v\left(k_{3}-k_{2} v+k_{3} v-u\right)$, with $-1 \leqslant v<0$, and

$$
\frac{d u}{d t}=a v-1-u^{2}+k_{2} u, \quad \frac{d v}{d t}=v\left(k_{2}-u\right)
$$

with $v \leqslant-1$.

Note that systems (19) have no infinite singular points because $v \leqslant-1$. For systems (18) we have

Proposition 5. For systems (18) the following statements hold.

(I) If $\Delta_{3}<0$, then systems (18) have no infinite singular points.

(II) If $\Delta_{3}=0$, then systems (18) have one infinite singular point $E_{3}=\left(\frac{k_{3}}{2}, 0\right)$, and it is a saddle-node.

(III) If $\Delta_{3}>0$, then systems (18) have two infinite singular points: $E_{3}^{+}=$ $\left(\frac{k_{3}+\sqrt{\Delta_{3}}}{2}, 0\right)$, which is a saddle for $k_{3}>0$, and a stable node for $k_{3}<0$; $E_{3}^{-}=\left(\frac{k_{3}-\sqrt{\Delta_{3}}}{2}, 0\right)$, which is an unstable node for $k_{3}>0$, and a saddle for $k_{3}<0$.

Proof. The proof of this proposition is similar to the proof of Proposition 5, we omit it here.

2.4. Chart $\mathbf{U}_{2}$. Let $x=\frac{u}{v}, y=\frac{1}{v}, v>0$, then systems (4) satisfying (2) become

$$
\frac{d u}{d t}=1-\varepsilon a u v+\varepsilon u^{2}-k_{1} u, \quad \frac{d v}{d t}=\varepsilon v(u-a v),
$$

with $\frac{u}{v} \geqslant 1$, and

$$
\frac{d u}{d t}=1-\varepsilon a u v+\varepsilon u^{2}-k_{2} u, \quad \frac{d v}{d t}=\varepsilon v(u-a v),
$$

with $-1 \leqslant \frac{u}{v} \leqslant 1$, and

$$
\frac{d u}{d t}=1-\varepsilon a u v+\varepsilon u^{2}-k_{3} u-\left(k_{3}-k_{2}\right) v, \quad \frac{d v}{d t}=\varepsilon v(u-a v),
$$

with $\frac{u}{v} \leqslant-1$.

Since $(0,0)$ is not singular point of systems (20), (21) and (22), the origin of the local chart $U_{2}$ is not an infinite singular points. Consequently the origin of $V_{2}$ is not an infinite singular point. 
2.5. Chart $\mathbf{U}_{3}$. It is obvious that systems (4) satisfying (2) have a unique finite singular point $E_{2}=\left(a, k_{2} a\right)$. The local phase portrait of $E_{2}$ is described in the next result.

Proposition 6. Consider systems (4) satisfying (2) with $k_{2}<0$ and $-1<a<1$, then the following statements hold:

(I) If $\Delta_{2}<0$, then the finite singular point $E_{2}$ is an unstable focus.

(II) If $\Delta_{2} \geqslant 0$, then $E_{2}$ is an unstable node.

Note that focus and node are topologically equivalent, thus the topological type of the finite singular point $E_{2}$ is independent of $\Delta_{2}$. The proof of this result is straightforward and it is omitted.

\section{LiMit CYCLES}

This section is devoted to study the number of limit cycles for systems (4) satisfying (2).

First we introduce some definitions. A period annulus is a region in the plane completely filled by periodic orbits. A differential system has the radial angular monotonicity property (RAM property, for simplicity) if the differential system rotates monotonically along rays as the radius increases. A closed orbit surrounding the origin is called star like with respect to the origin if any rays through the origin intersect with the closed orbit in a unique point. The following lemma provides an useful criteria on the existence and uniqueness of a limit cycle for continuous differential systems, see [14].

Lemma 7. [Massera's Method] Consider continuous piecewise Liénard differential systems of the form

$$
\frac{d X}{d T}=F(X)-Y, \quad \frac{d Y}{d T}=G(X),
$$

with $X G(X)>0$ for $X \neq 0$, and that $F(0)=0$, so that the only singular point is at the origin. Assume that systems (23) have the RAM property and that they have no period annuli. If the systems have a periodic orbit then it is star-like with respect to the origin and it is a limit cycle which is unique and stable.

Using the results of [15] and Lemma 7, we can obtain the existence and uniqueness of limit cycles of systems (4) satisfying (2).

Proposition 8. For continuous piecewise Liénard differential systems (4) satisfying (2) with $-1<a<1$ and $k_{2}<0$, the following statements hold.

(I) A necessary condition for the existence of a limit cycle is that $k_{i}, i=1,2,3$ have not the same sign.

(II) If $k_{1}>0, k_{3}>0$, then the singular point $E_{2}$ is surrounded by a unique stable limit cycle.

(III) If $k_{1}<0, k_{3}>0$, then the singular point $E_{2}$ is surrounded by at most one limit cycle, which is unstable if it exist.

(IV) If $k_{1}>0, k_{3}<0$, then the singular point $E_{2}$ is surrounded by at most one limit cycle, which is stable if it exist. 
Proof. Doing the change of variables $X=x-a, Y=y-k_{2} a, T=-t$, then systems (4) satisfying (2) become

$$
\frac{d X}{d T}=F(X)-Y, \quad \frac{d Y}{d T}=X
$$

with

$$
F(X)= \begin{cases}k_{1}(X+a-1)+k_{2}(1-a) & \text { if } X \geqslant 1-a, \\ k_{2} X & \text { if }-(1+a) \leqslant X \leqslant 1-a, \\ k_{3}(X+1+a)-k_{2}(1+a) & \text { if } X \leqslant-(1+a) .\end{cases}
$$

We note that statements (I) and (II) of Proposition 8 follow as particular cases $r=l=1$ of statements (a) and (b) of Theorem 6 of [15] respectively. Since we have reverse the time, the stability of limit cycle has changed.

(III) According to statement (d) of Theorem 6 of [15], we just need to prove statement (III) of Proposition 8 for $k_{1}-k_{2}<0$. To show the nonnegative rotation property, we will compute the slope of the vector field along half-rays of the form $Y=\lambda X$. It is obvious that

$$
m_{\lambda}(X)=\left.\frac{d Y}{d X}\right|_{Y=\lambda X}=\frac{X}{F(X)-\lambda X} .
$$

In order to analyze the monotone character of this slope along the half-rays $Y=\lambda X$, we need to compute its derivative with respect to $X$, i.e.

$$
\frac{d m_{\lambda}(X)}{d X}=\frac{F(X)-X F^{\prime}(X)}{(F(X)-\lambda X)^{2}}
$$

0 .

If $-(1+a) \leqslant X \leqslant 1-a$, then $F(X)=k_{2} X$. Thus $\frac{d m_{\lambda}(X)}{d X}=\frac{\left(k_{2}-k_{2}\right) X}{(F(X)-\lambda X)^{2}}=$

If $X \geqslant 1-a$, then $F(X)=k_{1}(X+a-1)+k_{2}(1-a)$. It is obvious that $\frac{d m_{\lambda}(X)}{d X}=\frac{\left(k_{2}-k_{1}\right)(1-a)}{(F(X)-\lambda X)^{2}}>0$, because $k_{1}-k_{2}<0$ and $1-a>0$.

If $X \leqslant-(1+a)$, then $F(X)=k_{3}(X+1+a)-k_{2}(1+a)$. It follows that $\frac{d m_{\lambda}(X)}{d X}=\frac{\left(k_{3}-k_{2}\right)(1+a)}{(F(X)-\lambda X)^{2}}>0$, because $k_{3}>0, k_{2}<0$ and $1+a>0$.

According to Massera's Method, if $k_{1}<0, k_{3}>0$ and $k_{1}-k_{2}<0$, then the origin is surrounded by at most one limit cycle, and if it exists is stable.

For the case (IV) doing the change of variables $\bar{X}=-X$ and $\bar{Y}=-Y$, we have $\bar{k}_{2}=k_{2}>0, \bar{k}_{1}=k_{3}<0$ and $\bar{k}_{3}=k_{1}>0$. Then we can guarantee the uniqueness of limit cycles by statement (III).

Note that if either $k_{2}=k_{3}$, or $k_{1}=k_{2}$, then systems (4) satisfying (2) become continuous piecewise linear Liénard differential systems with two zones. Then according to Theorem 5 of [11] we have

Proposition 9. Consider continuous piecewise Liénard differential systems (4) satisfying (2) with $-1<a<1$ and $k_{2}<0$. Assume that $\Delta_{1}<0, \Delta_{3}<0$, then the following statements hold. 
PHASE PORTRAITS OF CONTINUOUS PIECEWISE LINEAR DIFFERENTIAL SYSTEMS 11

(I) If $k_{1}>0, k_{2}=k_{3}<0$ and $\frac{k_{1}}{\sqrt{-\Delta_{1}}}+\frac{k_{3}}{\sqrt{-\Delta_{3}}}>0$, then the singular point $E_{2}$ is surrounded by a unique stable limit cycle.

(II) If $k_{1}>0, k_{2}=k_{3}<0$ and $\frac{k_{1}}{\sqrt{-\Delta_{1}}}+\frac{k_{3}}{\sqrt{-\Delta_{3}}} \leqslant 0$, then the singular point $E_{2}$ is globally asymptotically unstable and no limit cycle exist.

(III) If $k_{1}=k_{2}>0, k_{3}<0$ and $\frac{k_{1}}{\sqrt{-\Delta_{1}}}+\frac{k_{3}}{\sqrt{-\Delta_{3}}} \geqslant 0$, then the singular point $E_{2}$ is surrounded by a unique unstable limit cycle.

(IV) If $k_{1}=k_{2}>0, k_{3}<0$ and $\frac{k_{1}}{\sqrt{-\Delta_{1}}}+\frac{k_{2}}{\sqrt{-\Delta_{2}}}<0$, then the singular point $E_{2}$ is globally asymptotically stable and no limit cycle exist.

Proof. First we consider the case $k_{1}>0, k_{2}=k_{3}<0$. Let $X=x-1, Y=$ $y-k_{2}, T=-t$, then systems (4) satisfying (2) become

$$
\frac{d X}{d T}=F(X)-Y, \quad \frac{d Y}{d T}=X-A,
$$

with $A=a-1<0$ and

$$
F(X)= \begin{cases}k_{1} X & \text { if } X \geqslant 0, \\ k_{2} X & \text { if } X \leqslant 0 .\end{cases}
$$

According with statements (a) and (b) of Theorem 5 of [11], we can prove statements (I) and (II), respectively.

Similar to the proofs of statements (I) and (II), we can deduce statements (III) and (IV) from statements (c) and (d) of Theorem 5 of [11].

\section{Proof of the RESUlts}

This section is devoted to prove our main results.

4.1. Proof of Theorem 1. The proof of Theorem 1 directly follows from the following result.

Proposition 10. The phase portraits of systems (4) satisfying (2) with $k_{1}<$ $0, k_{2}<0, k_{3}<0$ and $-1<a<1$ are topologically equivalent to

(I) Fig.1.1 if $\Delta_{1}<0, \Delta_{3}<0$;

(II) Fig.1.2 if either $\Delta_{1}=0, \Delta_{3}<0$, or $\Delta_{1}<0, \Delta_{3}=0$;

(III) Fig.1.3 if either $\Delta_{1}>0, \Delta_{3}<0$, or $\Delta_{1}<0, \Delta_{3}>0$;

(IV) Fig.1.4 if $\Delta_{1}=0, \Delta_{3}=0$;

(V) Fig. 1.5 if either $\Delta_{1}=0, \Delta_{3}>0$, or $\Delta_{1}>0, \Delta_{3}=0$;

(VI) Fig.1.6 if $\Delta_{1}>0, \Delta_{3}>0$.

Proof. Recall that the finite singular point $E_{2}$ is an unstable focus or node by Proposition 6, and there is no limit cycle by statement (I) of Proposition 8.

(I) For the case $\Delta_{1}<0, \Delta_{3}<0$, it is obvious that systems (4) satisfying (2) have no infinite singular points by statement (I) of Propositions 4 and 5 . Therefore 
the global phase portrait of systems (4) satisfying (2) is topologically equivalent to Fig.1.1.

(II) For the case $\Delta_{1}=0, \Delta_{3}<0$, the infinite singular point $E_{1}$ is a saddle-node by statement (II) of Proposition 4. Hence the global phase portrait of systems (4) satisfying (2) is topologically equivalent to Fig.1.2.

For the case $\Delta_{1}<0, \Delta_{3}=0$, the infinite singular point $E_{3}$ is a saddle-node by statement (II) of Proposition 5. So the global phase portrait of systems (4) satisfying (2) is topologically equivalent to Fig.1.2.

(III) For the case $\Delta_{1}>0, \Delta_{3}<0$, the infinite singular points $E_{1}^{+}$is a stable node, and $E_{1}^{-}$is a saddle by statement (III) of Proposition 4 . Then the global phase portrait of systems (4) satisfying (2) is topologically equivalent to Fig.1.3.

For the case $\Delta_{1}<0, \Delta_{3}>0$, the infinite singular points $E_{3}^{+}$is a stable node, and $E_{3}^{-}$is a saddle by statement (III) of Proposition 5 . Therefore the global phase portrait of systems (4) satisfying (2) is topologically equivalent to Fig.1.3.

(IV) For the case $\Delta_{1}=0, \Delta_{3}=0$, the infinite singular points $E_{1}$ and $E_{3}$ are saddle-nodes by statement (II) of Propositions 4 and 5 . Hence the global phase portrait of systems (4) satisfying (2) is topologically equivalent to Fig.1.4.

(V) For the case $\Delta_{1}>0, \Delta_{3}=0$, the infinite singular points $E_{1}^{+}$is a stable node, and $E_{1}^{-}$is a saddle by statement (III) of Proposition 4 , and the infinite singular point $E_{3}$ is a saddle-node by statement (II) of Propositions 5 . So the global phase portrait of systems (4) satisfying (2) is topologically equivalent to Fig.1.5.

For the case $\Delta_{1}=0, \Delta_{3}>0$, the infinite singular points $E_{3}^{+}$is a stable node, and $E_{3}^{-}$is a saddle by statement (III) of Proposition 5, and the infinite singular point $E_{1}$ is a saddle-node by statement (II) of Propositions 4 . Then the global phase portrait of systems (4) satisfying (2) is topologically equivalent to Fig.1.5.

(VI) For the case $\Delta_{1}>0, \Delta_{3}>0$, the infinite singular points $E_{1}^{+}$is a stable node, and $E_{1}^{-}$is a saddle by statement (III) of Proposition 4 . The infinite singular points $E_{3}^{+}$is a stable node, and $E_{3}^{-}$is a saddle by statement (III) of Proposition 5 . So the global phase portrait of systems (4) satisfying (2) is topologically equivalent to Fig.1.6.

4.2. Proof of Theorem 2. The proof of Theorem 2 directly follows from the following result.

Proposition 11. The phase portraits of systems (4) satisfying (2) with $k_{1}>$ $0, k_{2}<0, k_{3}>0$ and $-1<a<1$ are topologically equivalent to

(I) Fig.2.1 if $\Delta_{1}<0, \Delta_{3}<0$;

(II) Fig.2.2 if either $\Delta_{1}=0, \Delta_{3}<0$, or $\Delta_{1}<0, \Delta_{3}=0$;

(III) Fig.2.3 if either $\Delta_{1}>0, \Delta_{3}<0$, or $\Delta_{1}<0, \Delta_{3}>0$;

(IV) Fig.2.4 if $\Delta_{1}=0, \Delta_{3}=0$;

(V) Fig.2.5 if either $\Delta_{1}=0, \Delta_{3}>0$, or $\Delta_{1}>0, \Delta_{3}=0$;

(VI) Fig.2.6 if $\Delta_{1}>0, \Delta_{3}>0$. 
Proof. Recall that the finite singular point $E_{2}$ is an unstable focus or node by Proposition 6 , and there is a unique stable limit cycle by statement (II) of Proposition 8 .

(I) For the case $\Delta_{1}<0, \Delta_{3}<0$, it is obvious that systems (4) satisfying (2) have no infinite singular points by statement (I) of Propositions 4 and 5 . Then the global phase portrait of systems (4) satisfying (2) is topologically equivalent to Fig.2.1.

(II) For the case $\Delta_{1}=0, \Delta_{3}<0$, the infinite singular point $E_{1}$ is a saddle-node by statement (II) of Proposition 4 . Therefore the global phase portrait of systems (4) satisfying (2) is topologically equivalent to Fig.2.2.

For the case $\Delta_{1}<0, \Delta_{3}=0$, the infinite singular point $E_{3}$ is a saddle-node by statement (II) of Proposition 5. Hence the global phase portrait of systems (4) satisfying (2) is topologically equivalent to Fig.2.2.

(III) For the case $\Delta_{1}>0, \Delta_{3}<0$, the infinite singular points $E_{1}^{+}$is a saddle, and $E_{1}^{-}$is an unsaddle node by statement (III) of Proposition 4 . So the global phase portrait of systems (4) satisfying (2) is topologically equivalent to Fig.2.3.

For the case $\Delta_{1}<0, \Delta_{3}>0$, the infinite singular points $E_{3}^{+}$is a saddle, and $E_{3}^{-}$ is a stable node by statement (III) of Proposition 5. Then the global phase portrait of systems (4) satisfying (2) is topologically equivalent to Fig.2.3.

(IV) For the case $\Delta_{1}=0, \Delta_{3}=0$, the infinite singular points $E_{1}$ and $E_{3}$ are saddle-nodes by statement (II) of Propositions 4 and 5 . So the global phase portrait of systems (4) satisfying (2) is topologically equivalent to Fig.2.4.

(V) For the case $\Delta_{1}>0, \Delta_{3}=0$, the infinite singular points $E_{1}^{+}$is a saddle, and $E_{1}^{-}$is an unstable node by statement (III) of Proposition 4 , and the infinite singular point $E_{3}$ is a saddle-node by statement (II) of Propositions 5. Therefore the global phase portrait of systems (4) satisfying (2) is topologically equivalent to Fig.2.5.

For the case $\Delta_{1}=0, \Delta_{3}>0$, the infinite singular points $E_{3}^{+}$is a saddle, and $E_{3}^{-}$ is an unstable node by statement (III) of Proposition 5, and the infinite singular point $E_{1}$ is a saddle-node by statement (II) of Propositions 4 . Hence the global phase portrait of systems (4) satisfying (2) is topologically equivalent to Fig.2.5.

(VI) For the case $\Delta_{1}>0, \Delta_{3}>0$, the infinite singular points $E_{1}^{+}$is a saddle, and $E_{1}^{-}$is an unstable node by statement (III) of Proposition 4 . The infinite singular points $E_{3}^{+}$is a saddle, and $E_{3}^{-}$is an unstable node by statement (III) of Proposition 5. Then the global phase portrait of systems (4) satisfying (2) is topologically equivalent to Fig.2.6.

4.3. Proof of Theorem 3. The proof of Theorem 3 for the case $k_{1}>0, k_{2}<$ $0, k_{3}<0$ directly follows by the following result. For the case $k_{1}<0, k_{2}<0, k_{3}>0$, we can do the change of variables $X=-x$ and $Y=-y$.

Proposition 12. The phase portraits of systems (4) satisfying (2) with $k_{1}>$ $0, k_{2}<0, k_{3}<0$ and $-1<a<1$ are topologically equivalent to

(I) Fig.3.1 and Fig.3.2 if $\Delta_{1}<0, \Delta_{3}<0$; 
(II) Fig.3.3 if $\Delta_{1}=0, \Delta_{3}<0$;

(III) Fig.3.4 if $\Delta_{1}>0, \Delta_{3}<0$;

(IV) Fig.3.5 if $\Delta_{1}<0, \Delta_{3}>0$;

(V) Fig. 3.6 if $\Delta_{1}<0, \Delta_{3}=0$;

(VI) Fig.3.7, Fig.3.8, Fig. 3.9 and Fig. 3.10 if $\Delta_{1}>0, \Delta_{3}>0$;

(VII) Fig.3.11, Fig.3.12 and Fig.3.13 if $\Delta_{1}>0, \Delta_{3}=0$;

(VIII) Fig.3.14, Fig.3.15 and Fig.3.16 if $\Delta_{1}=0, \Delta_{3}>0$;

(IX) Fig.3.17, Fig.3.18, Fig.3.19 and Fig.3.20 if $\Delta_{1}=0, \Delta_{3}=0$.

In order to prove Proposition 12 we need the following result, for a proof see Theorem XVII of [20].

Theorem 13. Consider a heteroclinic loop with two singular points which are either two hyperbolic saddles, or one saddle and one saddle-node, with eigenvalues $\mu_{i} \leqslant 0 \leqslant \lambda_{i}$ for $i=1,2$ such that $\mu_{1} \mu_{2} \neq 0$ or $\lambda_{1} \lambda_{2} \neq 0$. Define $K=\left|\mu_{1} \mu_{2}\right| /\left(\lambda_{1} \lambda_{2}\right)$, if $K<1$ then the heteroclinic loop is stable, and if $K>1$ it is unstable.

Proof of Proposition 12. Recall that the finite singular point $E_{2}$ is an unstable focus or node by Proposition 6 , and there is at most one limit cycle by statement (IV) of Proposition 8.

(I) For the case $\Delta_{1}<0$ and $\Delta_{3}<0$, it is obvious that systems (4) satisfying (2) has no infinite singular points by statement (I) of Propositions 4 and 5 . In the following we will prove that systems (4) satisfying (2) can have no limit cycles and then they are topologically equivalent to Fig.3.1, or they have one limit cycle and then they are topologically equivalent to Fig.3.2.

We consider the special case $k_{2}=k_{3}<0$. From statements (II) of Proposition 9, if $\frac{k_{1}}{\sqrt{-\Delta_{1}}}+\frac{k_{3}}{\sqrt{-\Delta_{3}}} \leqslant 0$, then there is no limit cycles surrounding the singular point $E_{2}$. Therefore the global phase portrait of systems (4) satisfying (2) is topologically equivalent to Fig.3.1.

If $\frac{k_{1}}{\sqrt{-\Delta_{1}}}+\frac{k_{3}}{\sqrt{-\Delta_{3}}}>0$, then the singular point $E_{2}$ is surrounded by a stable limit cycle. Then the global phase portrait of systems (4) satisfying (2) is topologically equivalent to Fig.3.2.

(II) For the case $\Delta_{1}=0, \Delta_{3}<0$, the infinite singular point $E_{1}$ is a saddle-node by statement (II) of Proposition 4. Hence the global phase portrait is topologically equivalent to Fig.3.3.

(III) For the case $\Delta_{1}>0, \Delta_{3}<0$, the infinite singular points $E_{1}^{+}$is a saddle, and $E_{1}^{-}$is an unstable node by statement (III) of Proposition 4. So the global phase portrait is topologically equivalent to Fig.3.4.

(IV) For the case $\Delta_{1}<0, \Delta_{3}>0$, the infinite singular points $E_{3}^{+}$is a stable node, and $E_{3}^{-}$is a saddle by statement (III) of Proposition 5. Then the global phase portrait is topologically equivalent to Fig.3.5.

(V) For the case $\Delta_{1}<0, \Delta_{3}=0$, the infinite singular point $E_{3}$ is a saddlenode by statement (II) of Proposition 5. Therefore the global phase portrait is topologically equivalent to Fig.3.6. 
PHASE PORTRAITS OF CONTINUOUS PIECEWISE LINEAR DIFFERENTIAL SYSTEMS 15

(VI) For the case $\Delta_{1}>0, \Delta_{3}>0$, the infinite singular points $E_{1}^{+}$is a saddle, and $E_{1}^{-}$is an unstable node by statement (III) of Proposition 4. The infinite singular points $E_{3}^{+}$is a stable node, and $E_{3}^{-}$is saddle node by statement (III) of Proposition 5 .

First we consider the subcase $\Delta_{2}<0$. The finite singular point $E_{2}$ is an unstable focus. Systems (4) satisfying (2) in the middle zone $-1 \leqslant x \leqslant 1$ have the first integral

$$
\begin{gathered}
H(x, y)=\exp ^{2 k_{2}^{2} \operatorname{ArcTan}\left(\frac{2 a+\left(k_{2}^{2}-2\right) x-k_{2} y}{\left(k_{2} x-y\right) \sqrt{-\Delta_{2}}}\right)}|x-a|^{2 k_{2} \sqrt{-\Delta_{2}}} \\
\quad \times\left(\frac{a^{2}+a\left(k_{2}^{2}-2\right) x+x^{2}-k_{2}(a+x) y+y^{2}}{(a-x)^{2}}\right) .
\end{gathered}
$$

Note that $H\left(a, k_{2} a\right)=+\infty$, so the first integral (30) is a decreasing function in a neighborhood of $E_{2}$.

Since $\Delta_{1}>0$ the finite singular point $\left(a, k_{1}(a-1)+k_{2}\right)$ is a virtual node with the stable invariant straight line

$$
\Gamma_{R}: \quad y=\frac{k_{1}+\sqrt{\Delta_{1}}}{2}(x-a)+k_{1}(a-1)+k_{2},
$$

which intersects the switching line $x=1$ at the point $\left(1, y_{1}\right)$, with

$$
y_{1}=\frac{(a-1) k_{1}+\sqrt{\Delta_{1}}}{2}+k_{2} \text {. }
$$

Since $\Delta_{3}>0$ the finite singular point $\left(a, k_{3}(a+1)-k_{2}\right)$ is a virtual node with the unstable invariant straight line

$$
\Gamma_{L}: \quad y=\frac{k_{3}-\sqrt{\Delta_{3}}}{2}(x-a)+k_{3}(a+1)-k_{2},
$$

which intersects the witching line $x=-1$ at the point $\left(-1, y_{2}\right)$, with

$$
y_{2}=\frac{(a+1) k_{3}+\sqrt{\Delta_{3}}}{2}-k_{2} \text {. }
$$

We define the auxiliary function

$$
\bar{H}(a)=H\left(1, y_{1}\right)-H\left(-1, y_{2}\right), \quad a \in(-1,1) .
$$

In the following we prove that $\bar{H}(a)$ given by (35) is an increasing function. Computing the derivative of $\bar{H}(a)$ with respect to $a$ we have that $\bar{H}^{\prime}(a)$ is equal to

$$
\begin{aligned}
& \frac{-2 k_{2} \sqrt{-\Delta_{2}}\left((1-a)^{2}\left(k_{1}-k_{2}\right)\left(k_{1}-\sqrt{\Delta_{1}}\right)\right)^{k_{2} \sqrt{-\Delta_{2}}}}{1-a} \exp { }^{2 k_{2}^{2} \operatorname{ArcTan}\left(\frac{4-k_{1} k_{2}+\sqrt{\Delta_{1}} k_{2}}{\left(-k_{1}+\sqrt{\Delta_{1}} \sqrt{-\Delta_{2}}\right)}\right)} \\
& +\frac{-2 k_{2} \sqrt{-\Delta_{2}}\left((1+a)^{2}\left(k_{3}-k_{2}\right)\left(k_{3}+\sqrt{\Delta_{3}}\right)\right)^{k_{2} \sqrt{-\Delta_{2}}}}{1+a} \exp 2 k_{2}^{2} \operatorname{ArcTan}\left(\frac{-4+k_{2} k_{3}+\sqrt{\Delta_{3}} k_{2}}{\left(k_{3}+\sqrt{\Delta_{3}} \sqrt{-\Delta_{2}}\right)}\right) .
\end{aligned}
$$

It is obvious that $\bar{H}^{\prime}(a)>0$. Note that $\lim _{a \rightarrow-1} \bar{H}(a)=-\infty$ and $\lim _{a \rightarrow 1} \bar{H}(a)=+\infty$, we can conclude that $\bar{H}(a)$ has a unique zero $a^{*}$ in the interval $a \in(-1,1)$. 
If $a<a^{*}$ then the separatrix $\Gamma_{R}$ of the saddle $E_{1}^{+}$is over the separatrix $\Gamma_{L}$ of the saddle $E_{3}^{-}$. According to Poincaré-Bendixson Theorem there is a stable limit cycle surrounding the finite singular point $E_{2}$. Hence the global phase portrait of systems (4) satisfying (2) is topologically equivalent to Fig.3.7.

If $a=a^{*}$ then there is a heteroclinic loop connecting $E_{1}^{+}$and $E_{3}^{-}$. In order to study the stability of this loop, we compute the eigenvalues of systems (12) and (18) at the singular point $E_{1}^{+}$and $E_{3}^{-}$, respectively. Then we obtain

$$
K=\left|\frac{\sqrt{\Delta_{1}}\left(k_{3}+\sqrt{\Delta_{3}}\right)}{\sqrt{\Delta_{3}}\left(k_{1}-\sqrt{\Delta_{1}}\right)}\right| .
$$

If $K>1$, then the loop is unstable by Theorem 13. According to PoincaréBendixson Theorem, there is a stable limit cycle surrounding the finite singular point $E_{2}$. Therefore the global phase portrait of systems (4) satisfying (2) is topologically equivalent to Fig.3.8.

If $K<1$, then the loop is stable by Theorem 13 . Hence the global phase portrait of systems (4) satisfying (2) is topologically equivalent to Fig.3.9.

If $a>a^{*}$, then the separatrix $\Gamma_{R}$ of the saddle $E_{1}^{+}$is under the separatrix $\Gamma_{L}$ of the saddle $E_{3}^{-}$. So the global phase portrait of systems (4) satisfying (2) is topologically equivalent to Fig.3.10.

Second we consider the subcase $\Delta_{2}=0$. Note that $k_{2}=-2$, then the finite singular point $E_{2}=(a,-2 a)$ is an unstable node with the invariant straight line $\Gamma_{C}=-x-a$, which intersects the switching line $x=-1$ at the point $\left(-1, \bar{y}_{L}\right)$ with $\bar{y}_{L}=1-a$, see Figure 5 . It is obvious that $y_{L}>\bar{y}_{L}$, thus the phase portrait of systems (4) satisfying (2) is topologically equivalent to Fig.3.10.

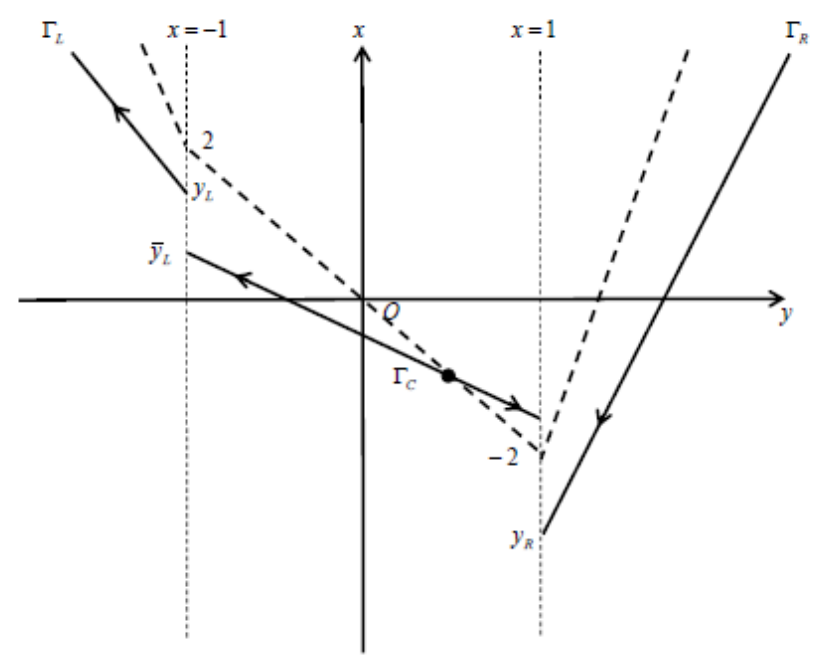

Figure 5. The invariant straight lines of systems (4) with $k_{1}>$ $0, k_{2}<0, k_{3}<0$ and $\Delta_{1}>0, \Delta_{2} \geqslant 0, \Delta_{3}>0$. 
Finally we consider the subcase $\Delta_{2}>0$. The finite singular point $E_{2}$ is an unstable node with the invariant straight line $\Gamma_{C}=\frac{k_{2}+\sqrt{\Delta_{2}}}{2}(x-a)+k_{2} a$, which intersects the switching line $x=-1$ at the point $\left(-1, \bar{y}_{L}\right)$ with $\bar{y}_{L}=$ $-\frac{k_{2}+\sqrt{\Delta_{2}}}{2}(1+a)+k_{2} a$. It is easy to deduce that $y_{L}>\bar{y}_{L}$ when $k_{2}<-2$. Thus the phase portrait of systems (4) satisfying (2) is topologically equivalent to Fig.3.10.

(VII) For the case $\Delta_{1}>0, \Delta_{3}=0$, the infinite singular points $E_{1}^{+}$is a saddle, $E_{1}^{-}$is an unstable node by statement (III) of Proposition 4, and the infinite singular point $E_{3}$ is a saddle-node by statement (II) of Propositions 5 . Note that $k_{3}=-2$, then $K=+\infty$. Similarly with the proof of statement (VI) we have:

If $a<a^{*}, \Delta_{2}<0$, then the global phase portrait of systems (4) satisfying (2) is topologically equivalent to Fig.3.11.

If $a=a^{*}, \Delta_{2}<0$, then the global phase portrait of systems (4) satisfying (2) is topologically equivalent to Fig.3.12.

If either $a>a^{*}, \Delta_{2}<0$, or $\Delta_{2} \geqslant 0$, then the global phase portrait of systems (4) satisfying (2) is topologically equivalent to Fig.3.13.

(VIII) For the case $\Delta_{1}=0, \Delta_{3}>0$, the infinite singular point $E_{1}$ is a saddlenode by statement (II) of Propositions 4 , the infinite singular points $E_{3}^{+}$is a stable node, and $E_{3}^{-}$is a saddle by statement (III) of Proposition 5 . Note that $k_{1}=2$, then $K=0$. Similarly with the proof of statement $(\mathrm{V})$ we have:

If $a<a^{*}, \Delta_{2}<0$, then the global phase portrait of systems (4) satisfying (2) is topologically equivalent to Fig.3.14.

If $a=a^{*}, \Delta_{2}<0$, then the global phase portrait of systems (4) satisfying (2) is topologically equivalent to Fig.3.15.

If either $a>a^{*}, \Delta_{2}<0$, or $\Delta_{2} \geqslant 0$, then the global phase portrait of systems (4) satisfying (2) is topologically equivalent to Fig.3.16.

(IX) For the case $\Delta_{1}=0, \Delta_{3}=0$, the infinite singular points $E_{1}$ and $E_{3}$ are two saddle-nodes by statement (II) of Propositions 4 and 5 . The proof of statement (IX) can be deduced from the proof of statement (V) we omit it here.

\section{Conclusion}

In this paper we classify the topological phase portraits in the Poincaré disc of the continuous piecewise Liénard differential systems (4) satisfying (2) with a unique singular point $E_{2}$ located at the middle zone. From the proofs of the three theorems which are given in section 4 , we can obtain the conditions for the existence and uniqueness of limit cycles for systems (4) satisfying (2). It is worth to point out that the amplitude of limit cycles is also very important, see for instance $[2,4,22]$.

For the case $k_{1}>0, k_{1}<0, k_{3}>0$, we obtain that systems (4) satisfying (2) have a unique stable limit cycle by Theorem 2. In fact we can proved that if $\Delta_{2} \geqslant 0$, then the limit cycle is contained in three zones. While if $\Delta_{2}<0$ and $1-a>0$ (resp. $a+1>0$ ) sufficiently small, then the limit cycle is contained in the middle 
and the right (resp. left) zones. These three cases have the same phase portrait according to the definition of topological equivalence given in section 1 .

For the case $k_{1}>0, k_{2}<0, k_{3}<0$, we know that the limit cycle of systems (4) satisfying (2) can be contained either in three zones, or in the middle and the right zones by numerical simulations. Unfortunately we cannot give an analytical proof because the computations are huge.

\section{Acknowledgements}

The first author is partially supported by the Natural Science Foundation of Guangdong Province (2017A030313010), Science and Technology Program of Guang zhou (No. 201707010426), National Natural Science Foundations of China (No. 11401111, 11661017) and China Scholarship Council (No. 201608440447).

The second author is partially supported by the MINECO-FEDER grant MTM 2016-77278-P, the AGAUR grant 2017 SGR1617, and the projecte MDM-2014-0445 (BGSMath).

\section{REFERENCES}

[1] D.C. Braga, L.F. Mello, Limit cycles in a family of discontinuous piecewise linear differential systems with two zones in the plane, Nonlinear Dyn. 73 (2003) 1283-1288.

[2] Y. Cao, C. Liu, The estimate of the amplitude of limit cycles of symmetric Liénard systems, J. Differential Equations 262 (2017) 2025-2038.

[3] P.T. Cardin, J. Torregrosa, Limit cycles in planar piecewise linear differential systems with nonregular separation line, Physica D 337 (2016) 67-82.

[4] M. Desroches, et al., Canards in piecewise-linear systems: explosions and super-explosions, Proc. R. Soc. A 469 (2012) 20120603-1-18.

[5] M. Desroches, et al., Canards, folded nodes, and mixed-mode oscillations in piecewise-linear slow-fast systems, SIAM Review 58 (2016) 653-691.

[6] F. Dumortier, J. Llibre, J. C. Artés, Qualitative Theory of Planar Differential Systems, Berlin Heidelberg: Springer-Verlag, 2006.

[7] R. Euzébio, R. Pazim, E. Ponce, Jump bifurcations in some degenerate planar piecewise linear differential systems with three zones, Physica D 325 (2016) 74-85.

[8] E. Freire, E. Ponce, F. Rodrigo, F. Torres, Bifurcation sets of continuous piecewise linear systems with two zones, Int. J. Bifurcation and Chaos 8 (1998) 2073-2097.

[9] E. Freire, E. Ponce, F. Rodrigo, F. Torres, Bifurcation sets of symmetric continuous piecewise linear systems with three zones, Int. J. Bifurcation and Chaos 12 (2002) 1675-1702.

[10] E. Freire, E. Ponce, J. Ros, Limit cycle bifurcation from center in symmetric piecewise-linear systems, Int. J. Bifurcation and Chaos 9 (1999) 895-907.

[11] E. Freire, E. Ponce, F. Torres, Hopf-like bifurcations in planar piecewise linear systems, Publicacions Matematiques 41 (1997) 135-148.

[12] M. Han, W. Zhang, On hopf bifurcation in non-smooth planar systems, J. Differential Equations 248 (2010) 2399-2416.

[13] J. Llibre, E. Nuñez, A.E. Teruel, Limit cycles for planar piecewise linear differential systems via first integrals, Quil. Theory Dyn. Syst. 3 (2002) 29-50.

[14] J. Llibre, M. Ordoñez, E. Ponce, On the existence and uniquess of limit cycles in planar continuous piecewise linear systems without symmetry, Nonlinear Analysis: Real World Applications 14 (2013) 2002-2012.

[15] J. Llibre, E. Ponce, C. Valls, Uniqueness and Non-uniqueness of limit cycles for piecewise linear differential systems with three zones and no symmetry, J. Nonlinear Sci. 25 (2015) $861-887$.

[16] J. Llibre, E. Ponce, X. Zhang, Existence of piecewise linear differential systems with exactly $n$ limit cycles for all $n \in N$, Nonlinear Analysis 54 (2003) 977-994. 
PHASE PORTRAITS OF CONTINUOUS PIECEWISE LINEAR DIFFERENTIAL SYSTEMS 19

[17] J. Llibre, A.E. Teruel, Introduction to the qualitative theory of differential systems, planar, symmetric and continuous piecewise linear systems, Birkhauser: Springer Basel, 2014.

[18] R. Lum, L. O. Chua, Global properties of continuous piecewise-linear vector fields. Part I: Simplest case in $\mathbb{R}^{2}$, Internat. J. Circuit Theory Appl. 19 (1991) 251-307.

[19] R. Lum, L. O. Chua, Global properties of continuous piecewise-linear vector fields. Part II: simplest symmetric in $\mathbb{R}^{2}$, Internat. J. Circuit Theory Appl. 20 (1992) 9-46.

[20] H. Poincaré, Mémoire sur les courbes définiés par une équation différentielle, J. de Math. Pures et appliquées, 3e série 7 (1881) 375-422.

[21] E. Ponce, J. Ros, E. Vela, Limit cycle and boundary equilibrium bifurcations in continuous planar piecewise linear systems, Int. J. Bifurcation and Chaos 25 (2015) 1530008-1-18.

[22] L. Yang, X. Zeng, An upper bound for the amplitude of limit cycles in Liénard systems with symmetry, J. Differential Equations 258 (2015) 2701-2710.

1 School of Mathematics and Statistics, Guangdong University of Finance and Economics, Guangzhou, 510320, P.R. China

E-mail address: lism19830126.com

2 Departament de Matemàtiques, Universitat Autònoma de Barcelona, 08193 Bellaterra, Barcelona, Catalonia, Spain

E-mail address: jllibre@mat.uab.cat 